\title{
Huygens' principle and iterative methods in inverse obstacle scattering
}

\author{
Olha Ivanyshyn • Rainer Kress • Pedro Serranho
}

Received: 15 April 2008 / Accepted: 22 June 2009 /

Published online: 22 July 2009

(C) The Author(s) 2009. This article is published with open access at Springerlink.com

\begin{abstract}
The inverse problem we consider in this paper is to determine the shape of an obstacle from the knowledge of the far field pattern for scattering of time-harmonic plane waves. In the case of scattering from a sound-soft obstacle, we will interpret Huygens' principle as a system of two integral equations, named data and field equation, for the unknown boundary of the scatterer and the induced surface flux, i.e., the unknown normal derivative of the total field on the boundary. Reflecting the ill-posedness of the inverse obstacle scattering problem these integral equations are ill-posed. They are linear with respect to the unknown flux and nonlinear with respect to the unknown boundary and offer, in principle, three immediate possibilities for their iterative solution via linearization and regularization. In addition to presenting new results on injectivity and dense range for the linearized operators, the main purpose of this paper is to establish and illuminate relations between these three solution methods based on Huygens' principle in inverse obstacle scattering. Furthermore, we will exhibit connections and differences to the
\end{abstract}

\footnotetext{
O. Ivanyshyn · R. Kress $(\varangle)$

Institut für Numerische und Angewandte Mathematik, Universität Göttingen, 37083 Göttingen, Germany

e-mail:kress@math.uni-goettingen.de

O. Ivanyshyn

e-mail: ivanyshy@math.uni-goettingen.de

P. Serranho

CEMAT, Instituto Superior Técnico,

Lisbon, Portugal

P. Serranho

Polytechnical Institute of Leiria,

2411-901 Leiria, Portugal

e-mail: pedro.serranho@estg.ipleiria.pt
}

Communicated by guest editors: Jin Cheng, Yiu Chung Hon and Masahiro Yamamoto. 
traditional regularized Newton type iterations as applied to the boundary to far field map, including alternatives for the implementation of these Newton iterations.

Keywords Inverse scattering $\cdot$ Nonlinear integral equations $\cdot$ Sound-soft obstacle Mathematics Subject Classifications (2000) 35J05 • 35R25 • 35R30 • 45Q05

\section{Introduction}

The scattering of a time-harmonic acoustic incident wave $u^{i}$ by a sound-soft obstacle $D$, that is, a bounded domain $D \subset \mathbb{R}^{m}$ for $m=2,3$ with a connected complement, is modeled by an exterior boundary value problem for the Helmholtz equation

$$
\Delta u^{s}+k^{2} u^{s}=0 \quad \text { in } \mathbb{R}^{m} \backslash \bar{D}
$$

with positive wave number $k$. The scattered wave $u^{s}$ has to satisfy the Dirichlet boundary condition

$$
u^{i}+u^{s}=0 \quad \text { on } \partial D
$$

and the Sommerfeld radiation condition

$$
\frac{\partial u^{s}}{\partial r}-i k u^{s}=o\left(\frac{1}{r^{\frac{m-1}{2}}}\right), \quad r=|x| \rightarrow \infty,
$$

uniformly for all directions. The total wave $u$ is obtained via superposition $u=$ $u^{i}+u^{s}$ and, for most of this paper we assume the incident wave to be a plane wave, that is,

$$
u^{i}(x, d)=e^{i k x \cdot d}
$$

where the unit vector $d$ is the direction of propagation.

Although most of our analysis has extensions to the impedance and/or the Neumann boundary condition, for the sake of exposition and simplicity, we have deliberately chosen to confine our presentation to the case of the Dirichlet boundary condition.

The Sommerfeld radiation condition characterizes outgoing waves and ensures uniqueness for the obstacle scattering problem (see [3]). For brevity, solutions $u^{s}$ to the Helmholtz equation that satisfy the Sommerfeld radiation condition are called radiating solutions. They can be shown to have an asymptotic behavior of the form

$$
u^{s}(x)=\frac{e^{i k|x|}}{|x|^{\frac{m-1}{2}}}\left\{u_{\infty}(\hat{x})+O\left(\frac{1}{|x|}\right)\right\}, \quad|x| \rightarrow \infty, \quad \hat{x}:=\frac{x}{|x|},
$$

uniformly with respect to all directions. The function $u_{\infty}$ is known as the far field pattern of the scattered wave and is an analytic function of $\hat{x}$ on the 
unit sphere $S^{m-1}:=\left\{x \in \mathbb{R}^{m}:|x|=1\right\}$. As one of the most important tools in scattering theory, Rellich's lemma (see Theorem 2.13 in [3]) provides a one-toone correspondence between a radiating solution $u^{s}$ to the Helmholtz equation and its far field pattern $u_{\infty}$ in the sense that $u_{\infty}=0$ on $S^{m-1}$ (or on an open subset of $S^{m-1}$ ) implies that $u^{s}=0$ in its domain of definition.

The inverse scattering problem that we are concerned with is to determine the shape and location of the scatterer $D$ from the knowledge of the far field pattern $u_{\infty}$ for one incident plane wave. We note that this inverse problem is nonlinear in the sense that the scattered wave depends nonlinearly on the scatterer $D$. More importantly, it is ill-posed since the determination of $D$ does not depend continuously on the far field pattern in any reasonable norm.

The question of uniqueness for the inverse scattering problem for soundsoft obstacles is not yet completely resolved. However, the far field pattern for one incident plane wave is sufficient to uniquely determine the obstacle $D$ if we have the a priori information that the diameter of $D$ is less than $2 c_{m} / k$ where $c_{m}$ is the smallest positive zero of the Bessel function $J_{m / 2}$ of order $m / 2$, see $[5,10]$. For detailed presentations on inverse obstacle scattering including the issue of uniqueness we refer to the monographs $[1,3,33]$ and the surveys $[2$, $4,26,35,36]$.

Here, for the foundation of a class of iterative reconstruction methods we start from Green's representation for the scattered wave for a sound-soft obstacle

$$
u^{s}(x)=-\int_{\partial D} \frac{\partial u}{\partial v}(y) \Phi(x, y) d s(y), \quad x \in \mathbb{R}^{m} \backslash \bar{D},
$$

in terms of the fundamental solution

$$
\Phi(x, y)=\left\{\begin{array}{l}
\frac{i}{4} H_{0}^{(1)}(k|x-y|), \quad x \neq y, \quad m=2, \\
\frac{e^{i k|x-y|}}{4 \pi|x-y|}, \quad x \neq y, \quad m=3,
\end{array}\right.
$$

where $H_{0}^{(1)}$ denotes the Hankel function of the first kind and order zero (see Theorem 3.12 in [3]). The unit normal vector $v$ to $\partial D$ is assumed to be directed into the exterior of $D$. The far field pattern of (1.5) is given by

$$
u_{\infty}(\hat{x})=-\gamma_{m} \int_{\partial D} \frac{\partial u}{\partial v}(y) e^{-i k \hat{x} \cdot y} d s(y), \quad \hat{x} \in S^{m-1},
$$

where

$$
\gamma_{m}=\left\{\begin{array}{l}
\frac{e^{i \frac{\pi}{4}}}{\sqrt{8 \pi k}}, \quad m=2 \\
\frac{1}{4 \pi}, \quad m=3
\end{array}\right.
$$


The representations (1.5) and (1.6) of the scattered field and its far field pattern in terms of its secondary sources on the boundary is known as Huygens' principle.

In view of the sound-soft boundary condition (1.2) from (1.5) we conclude that

$$
u^{i}(x)=\int_{\partial D} \frac{\partial u}{\partial v}(y) \Phi(x, y) d s(y), \quad x \in \partial D .
$$

Now we can interpret Huygens' principle, that is, (1.6) and (1.7) as a system of two integral equations for the unknown boundary $\partial D$ of the scatterer and the induced surface flux

$$
\varphi:=-\frac{\partial u}{\partial v} \text { on } \partial D .
$$

Although, in principle, we are not interested in the surface flux in methods based on the simultaneous solution of (1.6) and (1.7) we cannot avoid solving also for $\varphi$. It is convenient to call (1.6) the data equation since it contains the given far field for the inverse problem and (1.7) as the field equation since it represents the boundary condition. Both equations are linear with respect to the flux and nonlinear with respect to the boundary. Equation (1.6) is severely ill-posed whereas (1.7) is only mildly ill-posed.

Obviously we have three options for an iterative solution of (1.6) and (1.7). In the first method, given a current approximation for the boundary $\partial D$ we can solve the mildly ill-posed integral equation of the first kind (1.7) for $\varphi$. Then, keeping $\varphi$ fixed we linearize the equation (1.6) with respect to $\partial D$ to update the boundary approximation. This approach has been suggested by Johansson and Sleeman [23] and analyzed further by Ivanyshyn and Johansson [19, 20]. In the second method, following ideas first developed for the Laplace equation by Kress and Rundell [28], one also can solve the system (1.6) and (1.7) simultaneously for $\partial D$ and $\varphi$ by Newton iterations, that is, by linearizing both equations with respect to both unknowns. This approach has been intensively studied by Ivanyshyn, Johansson and Kress [15-18, 21, 22]. Whereas in the first method the burden of the ill-posedness and nonlinearity is put on one equation, in the third method a more even distribution of the difficulties is obtained by reversing the roles of (1.6) and (1.7), that is, by solving the severely ill-posed equation (1.6) for $\varphi$ and linearize (1.7) for gaining the boundary update. In a slight modification, this approach may be interpreted also as a decomposition method since to some extent it separates the illposedness and the nonlinearity. It combines the traditional decomposition method with elements of Newton iterations. Therefore it has also been termed as a hybrid method and as such analyzed in a series of papers by Kress and Serranho [27, 29, 30, 37-39].

The paper is organized as follows. In Section 2 we present parameterized versions of the integral operators in the data and the field equations and their Fréchet derivatives with respect to the boundary shape. Then, in Subsection 3.1 
we discuss the first method, i.e., the iterative scheme obtained via solving the well-posed linear field equation for the flux and updating the boundary via linearization of the data equation. We generalize a result on injectivity and dense range of the linearized operator from the two-dimensional to the three-dimensional case. Further, we establish a new relation of this method with the traditional Newton iterations for the boundary to far field map. In Subsection 3.2 we proceed with investigating the second method, i.e., the simultaneous linearization of both the data and the field equation with respect to both unknowns. Again we present a new connection between this approach and the Newton iterations for the boundary to far field map. From this we deduce a result on injectivity and dense range for the two-by-two system of the linearized equations. In addition, we illustrate the use of the connection as an alternative approach for implementing the Newton iterations for the boundary to far field map. In Section 4 we finally consider the third method, i.e., first solving the ill-posed linear data equation for the flux and then updating the boundary by linearizing the field equation. In particular, we exhibit both the relation and the difference to the so-called hybrid method as suggested by Kress and Serranho and conclude with some numerical examples for reconstructions in three dimensions.

\section{Parameterized operators and derivatives}

In order to define the operators occurring in (1.6) and (1.7) rigorously, for ease of presentation, we restrict ourselves to boundaries $\partial D$ that can be parameterized through mapping them globally onto the unit sphere $S^{m-1}$, that is,

$$
\partial D=\left\{p(\hat{x}): \hat{x} \in S^{m-1}\right\}
$$

for some injective $C^{\infty}$ function $p: S^{m-1} \rightarrow \mathbb{R}^{m}$. As simple example, the reader should consider the case of starlike domains, where

$$
p(\hat{x})=r(\hat{x}) \hat{x}, \quad \hat{x} \in S^{m-1},
$$

with a radial distance function $r: S^{m-1} \rightarrow(0, \infty)$. Now, we introduce the parameterized single-layer operator and far field operator

$$
A, A_{\infty}: C^{2}\left(S^{m-1}, \mathbb{R}^{m}\right) \times L^{2}\left(S^{m-1}, \mathbb{C}\right) \rightarrow L^{2}\left(S^{m-1}, \mathbb{C}\right)
$$

by

$$
A(p, \psi)(\hat{x}):=\int_{S^{m-1}} \Phi(p(\hat{x}), p(\hat{y})) \psi(\hat{y}) d s(\hat{y}), \quad \hat{x} \in S^{m-1}
$$

and

$$
A_{\infty}(p, \psi)(\hat{x}):=\gamma_{m} \int_{S^{m-1}} e^{-i k \hat{x} \cdot p(\hat{y})} \psi(\hat{y}) d s(\hat{y}), \quad \hat{x} \in S^{m-1}
$$


Then the (1.6) and (1.7) can be written in the operator form

$$
A_{\infty}(p, \psi)=u_{\infty}
$$

and

$$
A(p, \psi)=-u^{i} \circ p
$$

where we have incorporated the surface element into the density function via

$$
\psi(\hat{x}):=J(\hat{x}) \varphi(p(\hat{x}))
$$

with the Jacobian $J$ of the mapping $p$. The linearization of these equations requires the Fréchet derivatives of the operators $A$ and $A_{\infty}$ with respect to $p$. According to [32], these can be obtained by formally differentiating their kernels with respect to $p$, that is,

$$
A^{\prime}(p, \psi ; q)(\hat{x})=\int_{S^{m-1}} \operatorname{grad}_{x} \Phi(p(\hat{x}), p(\hat{y})) \cdot[q(\hat{x})-q(\hat{y})] \psi(\hat{y}) d s(\hat{y})
$$

and

$$
A_{\infty}^{\prime}(p, \psi ; q)(\hat{x})=-i k \gamma_{m} \int_{S^{m-1}} e^{-i k \hat{x} \cdot p(\hat{y})} \hat{x} \cdot q(\hat{y}) \psi(\hat{y}) d s(\hat{y})
$$

for $\hat{x} \in S^{m-1}$. The operators $A^{\prime}$ and $A_{\infty}^{\prime}$ are linear with respect to $q$ and they represent linearizations in the sense that

$$
\left\|A(p+q, \psi)-A(p, \psi)-A^{\prime}(p, \psi ; q)\right\|_{L^{2}\left(S^{m-1}\right)}=o\left(\|q\|_{C^{2}\left(S^{m-1}\right)}^{2}\right)
$$

and

$$
\left\|A_{\infty}(p+q, \psi)-A_{\infty}(p, \psi)-A_{\infty}^{\prime}(p, \psi ; q)\right\|_{L^{2}\left(S^{m-1}\right)}=o\left(\|q\|_{C^{2}\left(S^{m-1}\right)}^{2}\right) .
$$

\section{Iterative solution of the integral equations}

\subsection{Linearization of the data equation}

Johansson and Sleeman [23] suggested the following iterative method for approximately solving the system (2.4) and (2.5) for the two-dimensional case. For fixed $p$, provided $k^{2}$ is not a Dirichlet eigenvalue of the negative Laplacian in $D$, both in a Hölder space setting $A(p, \cdot): C^{0, \alpha}\left(S^{m-1}\right) \rightarrow C^{1, \alpha}\left(S^{m-1}\right)$ or in a Sobolev space setting $A(p, \cdot): H^{-1 / 2}\left(S^{m-1}\right) \rightarrow H^{1 / 2}\left(S^{m-1}\right)$, the operator $A(p, \cdot)$ is a homeomorphism (see [3]). In this case, given an approximation to the boundary parameterization $p$ we can solve the field equation (2.5) for the density $\psi$. Then, keeping $\psi$ fixed, we linearize the data equation (2.4) with respect to $p$ to obtain the linear equation

$$
A_{\infty}^{\prime}\left(p,[\underbrace{[A(p, \cdot)]^{-1}\left(u^{i} \circ p\right)}_{-\psi} ; q)=-u_{\infty}-A_{\infty}(p, \underbrace{[A(p, \cdot)]^{-1}\left(u^{i} \circ p\right)}_{-\psi})\right.
$$


for $q$ to update the parameterization $p$ via $p+q$. This procedure is repeated until some stopping criterium is satisfied.

In principle, the parameterization of the update

$$
\text { ว } D_{q}=\left\{p(\hat{x})+q(\hat{x}): \hat{x} \in S^{m-1}\right\}
$$

is not unique. To cope with the ambiguity, the simplest possibility is to allow only perturbations of the form

$$
q=z v \circ p
$$

with a scalar function $z$ defined on the unit sphere $S^{m-1}$ and denote the corresponding linear space of normal $L^{2}$ vector fields by $L_{\text {normal }}^{2}\left(S^{m-1}\right)$.

For fixed $p$ the operator $A_{\infty}^{\prime}\left(p,[A(p, \cdot)]^{-1}\left(u^{i} \circ p\right) ; \cdot\right)$ has a smooth kernel and therefore is severely ill-posed. This requires stabilization, for example, via Tikhonov regularization. The following result ensures injectivity and dense range as prerequesits for Tikhonov regularization. It generalizes results from [17], were only the two-dimensional case was considered.

Theorem 3.1 Assume that $k^{2}$ is not a Neumann eigenvalue of the negative Laplacian in $D$. Then the operator

$$
A_{\infty}^{\prime}\left(p,[A(p, \cdot)]^{-1}\left(u^{i} \circ p\right) ; \cdot\right): L_{\text {normal }}^{2}\left(S^{m-1}\right) \rightarrow L^{2}\left(S^{m-1}\right)
$$

is injective and has dense range.

Proof We abbreviate $\psi:=-[A(p, \cdot)]^{-1}\left(u^{i} \circ p\right)$ and assume that $q=z \vee \circ p$ satisfies $A_{\infty}^{\prime}(p, \psi ; q)=0$. Then, from (2.8) we observe that the double-layer potential

$$
v(x)=\int_{S^{m-1}} v(p(\hat{y})) \cdot \operatorname{grad}_{x} \Phi(x, p(\hat{y})) \psi(\hat{y}) z(\hat{y}) d s(\hat{y})
$$

has vanishing far field pattern. Hence, by Rellich's lemma we can conclude that $v$ vanishes outside of $D$. By the $L^{2}$ jump relations for double-layer potentials together with the Fredholm alternative in dual systems it can be concluded that $z$ is continuous (see the proof of Theorem 5.5 in [3]). This in turn, by the jump relations, implies that $v$ solves the homogeneous Neumann problem in $D$ and therefore vanishes identically in $D$ by our assumption on $k$. A third application of the jump relations yields $\psi z=0$ on $S^{m-1}$. However, by Holmgren's theorem $\psi=-[A(p, \cdot)]^{-1}\left(u^{i} \circ p\right)$ cannot vanish on open subsets of $S^{m-1}$, since it represents the normal derivative $\partial u / \partial v$ of the total field $u$ for scattering of the plane wave $u^{i}$ from $D$. Consequently, we have $z=0$ on $S^{m-1}$.

Now, let $g$ be a solution to the homogeneous adjoint equation

$$
v(p(\hat{y})) \cdot \int_{S^{m-1}} \hat{x} e^{i k \hat{x} \cdot p(\hat{y})} \psi(\hat{y}) g(\hat{x}) d s(\hat{x})=0, \quad \hat{y} \in S^{m-1} .
$$


Then, keeping in mind that $\psi$ cannot vanish on open subsets of $S^{m-1}$, this implies that the Herglotz wave function

$$
w(y):=\int_{S^{m-1}} e^{i k \hat{x} \cdot y} g(\hat{x}) d s(\hat{x}), \quad y \in \mathbb{R}^{m},
$$

has a vanishing normal derivative on $\partial D$. By the assumption of the theorem on $k$, this implies that $w$ vanishes identically in $D$ and by analyticity $w$ vanishes everywhere. Therefore, the kernel $g$ of the Herglotz wave function must be identically zero (see Theorem 3.15 in [3]).

We can relate the above approach to traditional Newton iterations for solving the inverse obstacle scattering problem. Denoting by $F: p \rightarrow u_{\infty}$ the operator that maps the boundary $\partial D$ represented by the parameterization $p$ onto the far field pattern for scattering of the incident wave $u^{i}$ from $D$, the inverse problem is equivalent to solving the nonlinear operator equation

$$
F(p)=u_{\infty}
$$

With the above notations, in the case when $k^{2}$ is not a Dirichlet eigenvalue of the negative Laplacian in $D$, we can represent

$$
F(p)=-A_{\infty}\left(p,[A(p, \cdot)]^{-1}\left(u^{i} \circ p\right)\right) .
$$

By the product and chain rule this implies the Fréchet derivative

$$
\begin{aligned}
F^{\prime}(p ; q)= & -A_{\infty}^{\prime}\left(p,[A(p, \cdot)]^{-1}\left(u^{i} \circ p\right) ; q\right) \\
& +A_{\infty}\left(p,[A(p, \cdot)]^{-1} A^{\prime}\left(p,[A(p, \cdot)]^{-1}\left(u^{i} \circ p\right) ; q\right)\right) \\
& -A_{\infty}\left(p,[A(p, \cdot)]^{-1}\left(\left(\operatorname{grad} u^{i}\right) \circ p\right) \cdot q\right) .
\end{aligned}
$$

Hence, we have established a new interrelation between the iterative scheme of Johannson and Sleeman and the traditional Newton iterations for the boundary to far field map as expressed by the following theorem.

Theorem 3.2 The iteration scheme as given through (3.1) can be interpreted as Newton iterations for (3.3) with the derivate of $F$ approximated through the first term in the representation (3.5) only, which corresponds to keeping $\psi=-[A(p, \cdot)]^{-1}\left(u^{i} \circ p\right)$ fixed in the linearization process.

The iteration scheme given through (3.1) has been successfully numerically implemented in two dimensions (see $[18,23]$ ). As to be expected from the close relations to Newton iterations for (3.3) as just pointed out, the quality of the reconstructions can compete with those of Newton iterations with the benefit of reduced computational costs.

The above method of linearizing the data equation has also been extended to the case of sound-hard obstacles [20] in two dimensions. 
3.2 Simultaneous linearization of both equations

Extending a method proposed by Kress and Rundell [28] for an inverse Dirichlet problem for the Laplace equation, a second approach for iteratively solving the system (2.4) and (2.5) consists in simultaneously linearizing both equations with respect to both unknowns. In this case, given approximations $p$ and $\psi$ both for the boundary parameterization and the density we obtain the system of linear equations

$$
A_{\infty}^{\prime}(p, \psi ; q)+A_{\infty}(p, \chi)=-A_{\infty}(p, \psi)+u_{\infty}
$$

and

$$
A^{\prime}(p, \psi ; q)+\left(\left(\operatorname{grad} u^{i}\right) \circ p\right) \cdot q+A(p, \chi)=-A(p, \psi)-u^{i} \circ p .
$$

This system has to be solved for $q$ and $\chi$ in order to obtain updates $p+q$ for the boundary parameterization and $\psi+\chi$ for the density. This procedure is repeated until some stopping criterium is satisfied.

Again we restrict the updates to normal fields of the form (3.2) and note that due to the smoothness of the kernels both (3.6) and (3.7) are severely ill-posed and require regularization with respect to both variables. In particular, for the parameterization update it is appropriate to incorporate penalties for Sobolev norms of $q$ to guarantee smoothness of the boundary, whereas for the density $L^{2}$ penalty terms on $\chi$ are sufficient.

The simultaneous iterations (3.6) and (3.7) again exhibit relations to the Newton iteration

$$
F^{\prime}(p ; q)=u_{\infty}-F(p)
$$

obtained by linearization of (3.3). We present these new insights in the structure of iterative methods in inverse obstacle scattering by the following theorem.

Theorem 3.3 Assume that $k^{2}$ is not a Dirichlet eigenvalue of the negative Laplacian in $D$ and set $\psi:=-[A(p, \cdot)]^{-1}\left(u^{i} \circ p\right)$. Provided $q$ satisfies the linearized boundary to far field equation (3.8) then $q$ and

$$
\chi:=-[A(p, \cdot)]^{-1}\left(A^{\prime}(p, \psi ; q)+\left(\left(\operatorname{grad} u^{i}\right) \circ p\right) \cdot q\right)
$$

satisfy the linearized data and field equations (3.6) and (3.7). Conversely, if $q$ and $\chi$ solve (3.6) and (3.7) then $q$ satisfies (3.8).

Proof From (3.4) and the definition of $\psi$ we have

$$
F^{\prime}(p ; q)=u_{\infty}-A_{\infty}(p, \psi) .
$$

The representation (3.5) of the derivative of $F$ yields

$$
F^{\prime}(p, q)=A_{\infty}^{\prime}(p, \psi ; q)+A_{\infty}(p, \chi)
$$


and combining this with the previous equation establishes that (3.6) holds. From the definition of $\chi$ we observe

$$
\left.A(p, \chi)+A^{\prime}(p, \psi ; q)+\left(\left(\operatorname{grad} u^{i}\right) \circ p\right) \cdot q\right)=0
$$

Therefore, in view of $A(p, \psi)=-u^{i} \circ p$ we also have (3.7) satisfied.

Conversely, the second equation (3.7) implies that

$$
\chi=-[A(p, \cdot)]^{-1}\left(A^{\prime}(p, \psi ; q)+\left(\left(\operatorname{grad} u^{i}\right) \circ p\right) \cdot q\right)
$$

and inserting this into (3.6) leads to

$$
\begin{aligned}
A_{\infty}^{\prime}(p, \psi ; q) & -A_{\infty}\left(p,[A(p, \cdot)]^{-1}\left(A^{\prime}(p, \psi ; q)+\left(\left(\operatorname{grad} u^{i}\right) \circ p\right) \cdot q\right)\right) \\
& =-A_{\infty}(p, \psi)+u_{\infty}
\end{aligned}
$$

and via (3.5) this implies (3.8).

Based on Theorem 3.3 known results (see $[3,25]$ ) on injectivity and dense range of the derivative $F^{\prime}$ can be carried over to the system (3.6) and (3.7) by the following corollary.

Corollary 3.4 At the exact solution, the system (3.6) and (3.7) is injective and has dense range provided $k^{2}$ is neither a Dirichlet nor a Neumann eigenvalue of the negative Laplacian in $D$.

Remark 3.5 Theorem 3.3 also illustrates the difference between the iteration method based on (3.6) and (3.7) and the Newton iterations for (3.3). In general, when performing (3.6) and (3.7) in the sequence of updates the relation $A(p, \psi)=-\left(u^{i} \circ p\right)$ between the current approximations $p$ and $\psi$ for the parameterization and the density will not be satisfied. This observation also indicates a possibility to use (3.6) and (3.7) for implementing a Newton scheme for (3.3). We only need to change the update $\psi+\chi$ for the density by

$$
-[A(p+q, \cdot)]^{-1}\left(u^{i} \circ(p+q)\right)
$$

that is, at the expense of throwing away $\chi$ and solving a boundary integral equation for a new density.

In the literature, the implementation of Newton iterations for (3.3), in general, is based on the characterization of the Fréchet derivative of $F$ which is given by the far field $F^{\prime}(p ; q)=v_{\infty}$ of the radiating solution $v$ to the Helmholtz equation in the exterior of $D$ satisfying the Dirichlet condition

$$
v \circ q=-(\operatorname{grad} u) \circ q
$$


in terms of the total field $u$ (see Theorem 5.14 in [3]). For numerical implementations in three dimensions we refer to $[8,11]$.

The iteration scheme given through (3.6) and (3.7) has been numerically tested in two dimensions (see [21]). The performance of the method based on the linearization of the data equation from the previous subsection and the simultaneous linearization of this subsection, respectively, have been compared in [18]. In general, both methods work equally well with the following slight difference in strategy as observed from a large number of examples. The first method usually performs such that within the first iterations the initial guess is moved towards the center of the obstacle without much change in the shape and then first the illuminated part and then the shadow region of the obstacle is found. On the other hand, the second method searches right away for location and shape simultaneously. In some sense, this is to be expected since the far field pattern describes the asymptotic behavior of the scattered field and therefore is more sensible to the location of the scatterer than to its shape. Consequently, as the field equation is solved first and then (for a fixed flux density) the data equation is linearized, the first method first reconstructs the location and only later the shape. Since in the second method both the data and the field equation are linearized simultaneously, the search for location and shape also starts simultaneously. However, so far no deeper theoretical explanation for this behavior could be given.

Although there are a few results available on the convergence of regularized Newton iterations (see $[13,14,34]$ ) for inverse obstacle scattering problems, i.e., for the solution of (3.3) via (3.8), this issue is not satisfactorily resolved. Despite the progress made by Hohage $[13,14]$ with this respect, so far it has not been clarified whether the general results on the solution of ill-posed nonlinear equations in a Hilbert space setting (among others see $[6,7]$ ) are applicable to inverse obstacle scattering or, in general, to inverse boundary value problems in the frame work of solving the operator equation (3.3). The more problem oriented approach of Potthast [34] for a convergence analysis suffers from the restrictive assumption of a non-vanishing normal derivative of the total field on the boundary $\partial D$ in the case of exact data. Furthermore, in the analysis for noisy data, convergence for the noise level tending to zero, as usual, requires a stopping rule and with this particular rule the method has not yet been numerically implemented. These difficulties with establishing convergence results are also present in the iterations of this section, that is, currently the convergence issue remains an open problem.

The above method of simultaneous linearization has been extended to the case of sound-soft [22] and sound-hard [31] cracks in two dimensions. Here we note that as opposed to the hybrid method of the following section for crack reconstructions no additional penalty term on the length of the crack is required. In addition, it has been also applied to reconstructions of soundsoft or sound-hard scatterers from the modulus of the far field pattern [15]. In order to avoid the exceptional values occurring, for example, in Theorem 3.1 and 3.3 modifications using combined single- and double-layer potentials are suggested in [19]. 


\section{Decomposition methods revisited}

To evenly distribute the burden of the ill-posedness and the nonlinearity of the inverse obstacle scattering problem, instead of solving the field equation (2.5) for the density and then linearizing the data equation one can also think of solving the severely ill-posed data equation (2.4) for the density and linearize the only mildly ill-posed field equation to update the boundary. In this case, given an approximation for the boundary parameterization $p$ we first solve the data equation (2.4) for the density $\psi$. Then, keeping $\psi$ fixed, we linearize the field equation (2.5), that is, we obtain the linear equation

$$
A^{\prime}(p, \psi ; q)+\left(\left(\operatorname{grad} u^{i}\right) \circ p\right) \cdot q=-A(p, \psi)-u^{i} \circ p
$$

for $q$ to update the parameterization $p$ via $p+q$. Again, this procedure of alternatingly solving (2.4) and (4.1) must be repeated until some stopping criterium is satisfied. To some extent this procedure mimics a decomposition method in the sense that it decomposes the inverse problem into a severely ill-posed linear problem and an at most mildly ill-posed nonlinear problem.

To our knowledge, this approach has not been implemented numerically. However, the method that has been suggested and investigated by Kress and Serranho can be considered as a slight modification of the above procedure. In this method, given an approximation $p$ for the parameterization of the boundary, the data equation, that is,

$$
A_{\infty}(p, \psi)=u_{\infty}
$$

is solved for the density $\psi$ via regularization. Injectivity and dense range of the operator $A_{\infty}(p, \cdot): L^{2}\left(S^{m-1}\right) \rightarrow L^{2}\left(S^{m-1}\right)$ are guaranteed by Theorem 5.17 in [3] provided $k^{2}$ is not a Dirichlet eigenvalue for the negative Laplacian in $D$. Then we define the single-layer potential

$$
u^{s}(x)=\int_{S^{m-1}} \Phi(x, p(\hat{y})) \psi(\hat{y}) d s(\hat{y})
$$

and evaluate the boundary values of $u:=u^{i}+u^{s}$ and its derivatives on the surface represented by $p$ via the jump relations. Finally we find an update $p+$ $q$ for the boundary by linearizing the boundary condition $u \circ(p+q)=0$, that is, by solving the linear equation

$$
u \circ p+((\operatorname{grad} u) \circ p) \cdot q=0
$$

for $q$. In an obvious way, the two steps (4.2) and (4.3) are iterated. Again for uniqueness of the update representation we allow only perturbations of the form (3.2). Then, by Holmgren's theorem, injectivity for the linear equation (4.3) can be established at the exact boundary.

Since this approach combines ideas of the decomposition method due to Kirsch and Kress [24] with aspects of Newton iterations, it was termed a hybrid 
method. Convergence for exact data in the spirit of Potthast [34] and a stopping rule for noisy data were investigated in [38].

After introducing the operator

$$
\begin{aligned}
\widetilde{A}(p, \psi ; q)(\hat{x})= & \int_{S^{m-1}} \operatorname{grad}_{x} \Phi(p(\hat{x}), p(\hat{y})) \cdot q(\hat{x}) \psi(\hat{y}) d s(\hat{y}) \\
& -\frac{1}{2} \frac{\psi(\hat{x})[v(p(\hat{x})) \cdot q(\hat{x})]}{J(\hat{x})}
\end{aligned}
$$

and observing the jump relations for the single-layer potential and (2.6) we can rewrite (4.3) into the form

$$
\widetilde{A}(p, \psi ; q)+\left(\left(\operatorname{grad} u^{i}\right) \circ p\right) \cdot q=-A(p, \psi)-u^{i} \circ p .
$$

Comparing this with (4.1) we discover a new relation between solving the data and field equation iteratively via (2.4) and (4.1) and the hybrid method of Kress and Serranho.

Remark 4.1 In the hybrid method, the Fréchet derivative of $A$ with respect to $p$ is replaced by the operator $\widetilde{A}$ in the sense that we linearize only with respect to the evaluation surface for the single-layer potential but not with respect to the integration surface.

In Figs. 1, 2, 3, and 4 we present some examples for reconstructions by the above hybrid method. The ill-posed equation (2.4) is stabilized by Tikhonov
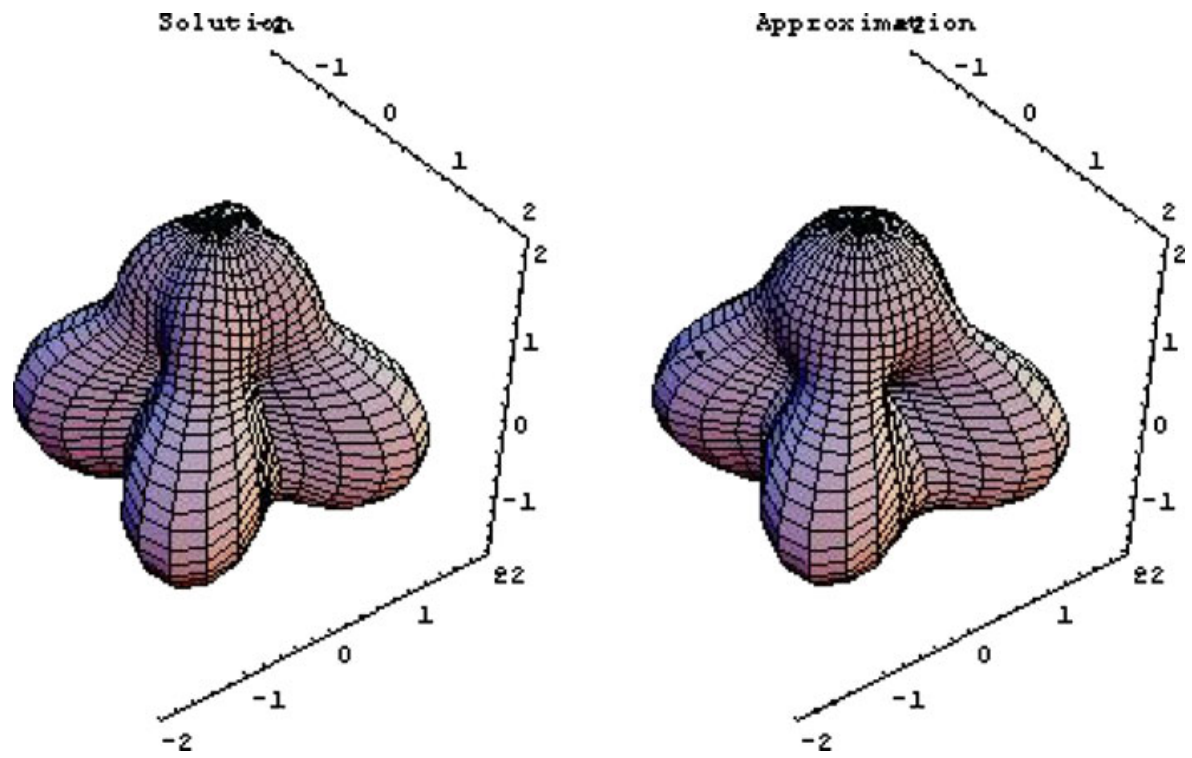

Fig. 1 Reconstruction of an acorn shaped domain 

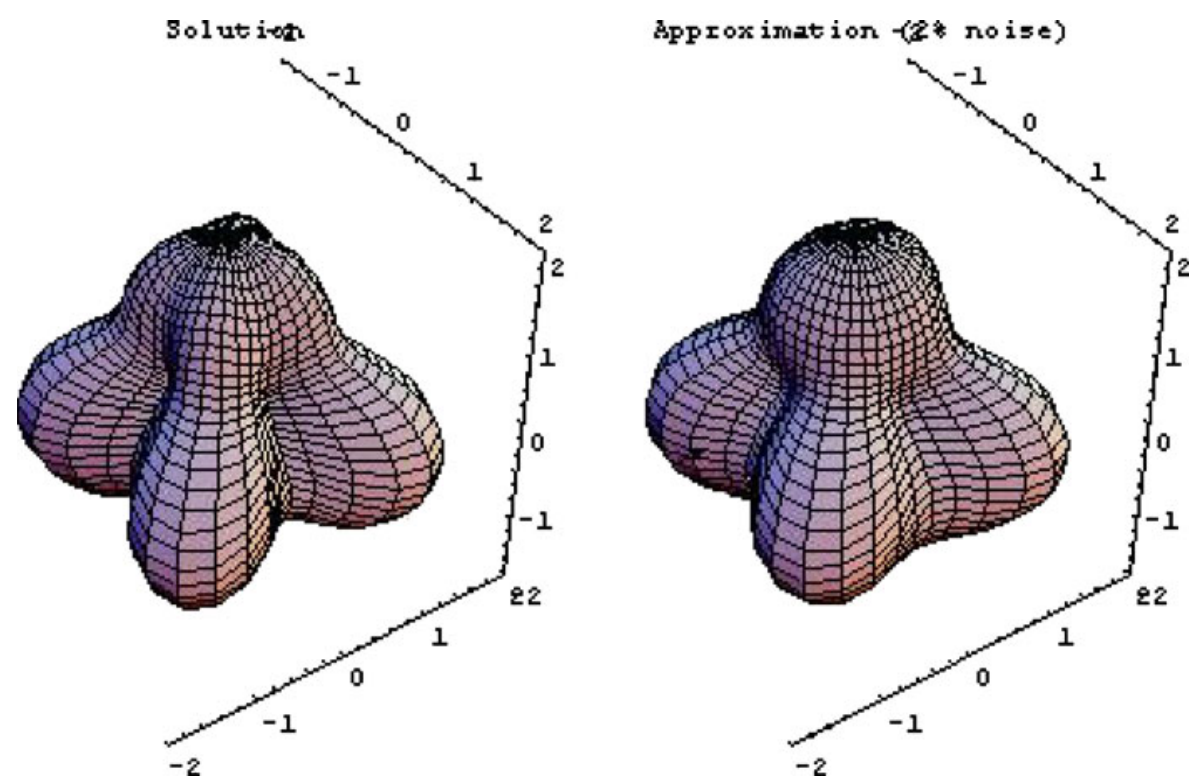

Fig. 2 Reconstruction of a pinched acorn shaped domain

regularization. The resulting integral equation of the second kind over the sphere $S^{2}$ is discretized via Nyström's method using the Gauss-trapezoidal rule with $N=164$ quadrature points. For the solution of (4.4), the radial distance
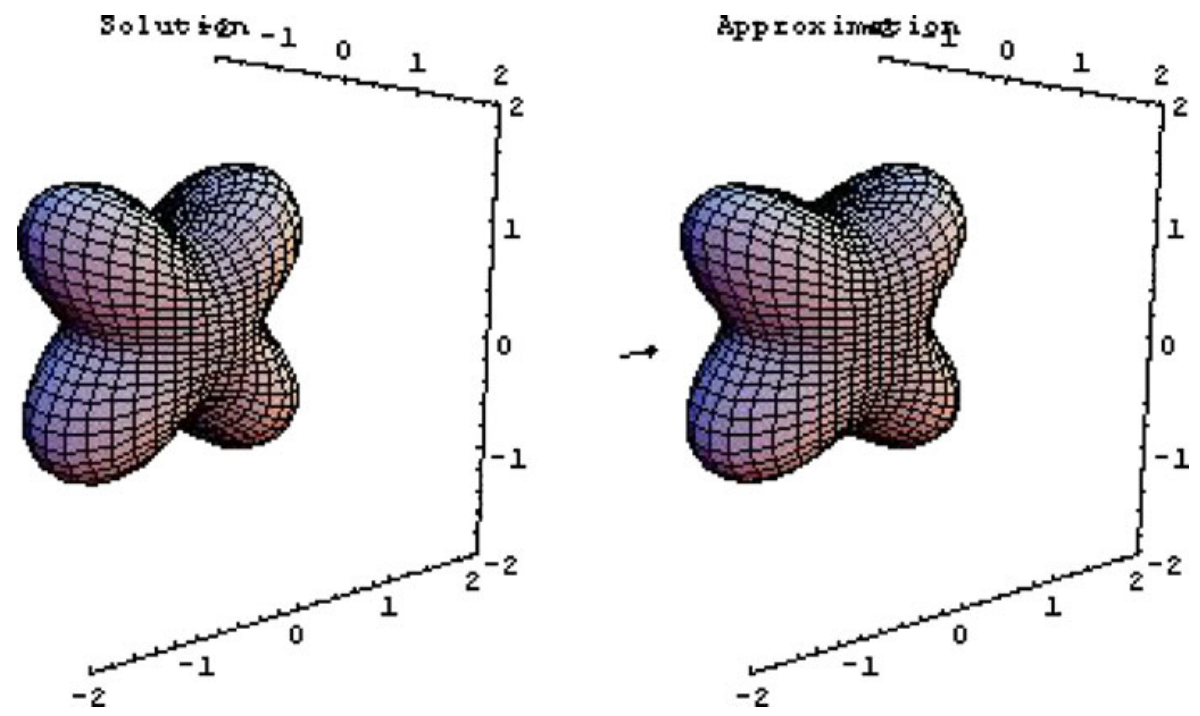

Fig. 3 Reconstruction of a star shaped domain 

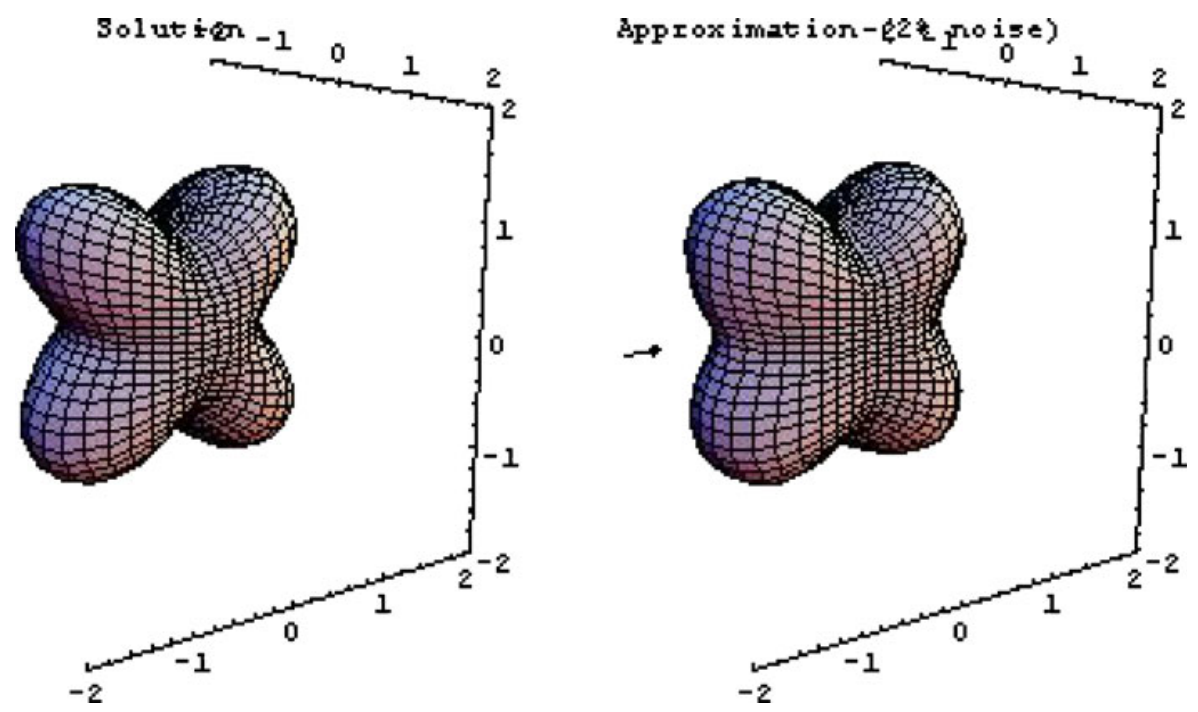

Fig. 4 Reconstruction of a cushion shaped domain

functions in the sense of (2.1) were approximated by linear combinations of real parts of spherical harmonics up to order eight which corresponds to 45 unknown coefficients. The linear system for these coefficients obtained by collocating (4.4) at $M=128$ points on $S^{2}$ was solved by a penalized least squares approach mimicking Tikhonov regularization. The numerical quadratures were based on Wienert's method [40] as described in Section 3.6 of [3] (see also [9]). This method can be viewed as a modification of the Gauss-trapezoidal rule for weakly singular integrals based on projections onto subspaces of spherical harmonics. In each example the figure on the left hand side gives the exact boundary shape and the figure on the right the reconstruction with one incident wave in direction of the arrow. The reconstructions are obtained with $2 \%$ random noise added to the synthetic far field pattern. The wave number is $k=1$.

This approach has been successfully extended to the case of sound-hard [30] and impedance obstacles [37]. For the impedance boundary condition both the shape and the impedance function can be reconstructed. In addition, the method has also been used for the reconstruction of sound-soft cracks in two dimensions [29]. In this case, it was necessary to impose a penalty term on the length of the crack. Otherwise, the algorithm would reconstruct cracks with length shrinking to zero.

Although the linearization of the homogeneous boundary Neumann condition (see [30]) is slightly more technical than the linearization of the homogeneous Dirichlet condition as described above, it is considerably less involved than the derivation of the boundary condition for the corresponding boundary to far field map $F$ for the sound hard case (see [12]). 


\section{Conclusion}

We have investigated and established new results on the interactions between three closely related methods for solving the inverse obstacle scattering problem via an iterative solution of nonlinear integral equations and illuminated their connections to Newton iterations for the boundary to far field map. These methods have been shown to be applicable for a range of boundary conditions and highly competitive with the Newton iterations. Their practical feasibility has been established in the literature through a variety of numerical examples.

Acknowledgements The research of O.I. was partially supported by the German Research Foundation DFG through the collaborative research center SFB 755. The research of P.S. was partially supported by the Calouste Gulbenkian Foundation.

Open Access This article is distributed under the terms of the Creative Commons Attribution Noncommercial License which permits any noncommercial use, distribution, and reproduction in any medium, provided the original author(s) and source are credited.

\section{References}

1. Cakoni, F., Colton, D.: Qualitative Methods in Inverse Scattering Theory: An Introduction. Springer, New York (2005)

2. Colton, D., Coyle, J., Monk, P.: Recent developments in inverse acoustic scattering theory. SIAM Rev. 42, 369-414 (2000)

3. Colton, D., Kress, R.: Inverse Acoustic and Electromagnetic Scattering Theory, 2nd edn. Springer, Berlin (1998)

4. Colton, D., Kress, R.: Using fundamental solutions in inverse scattering. Inverse Probl. 22, R49-R66 (2006)

5. Colton, D., Sleeman, B.D.: Uniqueness theorems for the inverse problem of acoustic scattering. IMA J. Appl. Math. 31, 253-259 (1983)

6. Deuflhard, P., Engl, H.W., Scherzer, O.: A convergence analysis of iterative methods for the solution of nonlinear ill-posed problems under affinely invariant conditions. Inverse Probl. 14, 1081-1106 (1998)

7. Engl, H.W., Hanke, M., Neubauer, A.: Regularization of Inverse Problems. Kluwer Academic, Dordrecht (1996)

8. Farhat, C., Tezaur, R., Djellouli, R.: On the solution of three-dimensional inverse obstacle acoustic scattering problems by a regularized Newton method. Inverse Probl. 18, 1229-1246 (2002)

9. Ganesh, M., Graham, I.: A high-order algorithm for obstacle scattering in three dimensions. J. Comput. Phys. 198, 211-242 (2004)

10. Gintides, D.: Local uniqueness for the inverse scattering problem in acoustics via the Faber-Krahn inequality. Inverse Probl. 21, 1195-1205 (2005)

11. Harbrecht, H., Hohage, T.: Fast methods for three-dimensional inverse obstacle scattering problems. J. Integral Equ. Appl. 19, 237-260 (2007)

12. Hettlich, F.: Frechet derivatives in inverse obstacle scattering. Inverse Probl. 14, 209-210 (1998)

13. Hohage, T.: Logarithmic convergence rates of the iteratively regularized Gauss-Newton method for an inverse potential and an inverse scattering problem. Inverse Probl. 13, 12791299 (1997)

14. Hohage, T.: Iterative methods in inverse obstacle scattering: regularization theory of linear and nonlinear exponentially ill-posed problems. Dissertation, Linz (1999)

15. Ivanyshyn, O.: Shape reconstruction of acoustic obstacles from the modulus of the far field pattern. Inverse Probl. Imaging 1, 609-622 (2007) 
16. Ivanyshyn, O.: Nonlinear boundary integral equations in inverse scattering. Dissertation Göttingen (2007)

17. Ivanyshyn, O., Johansson, T.: Boundary integral equations for acoustical inverse sound-soft scattering. J. Inverse Ill-Posed Probl. 15, 1-14 (2007)

18. Ivanyshyn, O., Johansson, T.: Nonlinear integral equation methods for the reconstruction of an acoustically sound-soft obstacle. J. Integral Equ. Appl. 19, 289-308 (2007)

19. Ivanyshyn, O., Johansson, T.: A coupled boundary integral equation method for inverse sound-soft scattering. In: Proceedings of Waves 2007. The 8th International Conference on Mathematical and Numerical Aspects of Waves, pp. 153-155. University of Reading (2007)

20. Ivanyshyn, O., Johansson, B.T.: Reconstruction of acoustically sound-hard obstacles from the far field using a boundary integral equation method. In: Charalambopoulos, A., Fotiadis, D.I., Polyzos, D. (eds.) Advanced Topics in Scattering and Biomedical Engineering, pp. 47-56 (2008)

21. Ivanyshyn, O., Kress, R.: Nonlinear integral equations in inverse obstacle scattering. In: Fotiatis, D.I., Massalas, C.V. (eds.) Mathematical Methods in Scattering Theory and Biomedical Engineering, pp. 39-50. World Scientific, Singapore (2006)

22. Ivanyshyn, O., Kress, R.: Inverse scattering for planar cracks via nonlinear integral equations. Math. Methods Appl. Sci. 31, 1221-1232 (2008)

23. Johansson, T., Sleeman, B.: Reconstruction of an acoustically sound-soft obstacle from one incident field and the far field pattern. IMA J. Appl. Math. 72, 96-112 (2007)

24. Kirsch, A., Kress, R.: An optimization method in inverse acoustic scattering. In: Brebbia, C.A., et al. (eds.) Boundary Elements IX. Fluid Flow and Potential Applications, vol. 3, pp. 3-18. Springer, Berlin (1987)

25. Kress, R.: Integral equation methods in inverse acoustic and electromagnetic scattering. In: Ingham, D.B., Wrobel, L.C. (eds.) Boundary Integral Formulations for Inverse Analysis, pp. 67-92. Computational Mechanics Publications, Southampton (1997)

26. Kress, R.: Acoustic scattering: scattering by obstacles. In: Pike, R., Sabatier, P. (eds.) Scattering, pp. 52-73. Academic, London (2001)

27. Kress, R.: Newton's method for inverse obstacle scattering meets the method of least squares. Inverse Probl. 19, 91-104 (2003)

28. Kress, R., Rundell, W.: Nonlinear integral equations and the iterative solution for an inverse boundary value problem. Inverse Probl. 21, 1207-1223 (2005)

29. Kress R., Serranho P.: A hybrid method for two-dimensional crack reconstruction. Inverse Probl. 21, 773-784 (2005)

30. Kress R., Serranho P.: A hybrid method for sound-hard obstacle reconstruction. J. Comput. Appl. Math. 204, 418-427 (2007)

31. Lee, Kuo-Ming: Inverse scattering via nonlinear integral equations for a Neumann crack Inverse Probl. 22, 1989-2000 (2006)

32. Potthast, R.: Fréchet differentiability of boundary integral operators in inverse acoustic scattering. Inverse Probl. 10, 431-447 (1994)

33. Potthast, R.: Point-Sources and Multipoles in Inverse Scattering Theory. Chapman \& Hall, London (2001)

34. Potthast, R.: On the convergence of a new Newton-type method in inverse scattering. Inverse Probl. 17, 1419-1434 (2001)

35. Potthast, R.: Sampling and probe methods-an algorithmical view. Computing 75, 215-235 (2005)

36. Potthast, R.: A survey on sampling and probe methods for inverse problems. Inverse Probl. 22, R1-R47 (2006)

37. Serranho, P.: A hybrid method for inverse scattering for shape and impedance. Inverse Probl. 22, 663-680 (2006)

38. Serranho, P.: A hybrid method for sound-soft obstacles in 3D. Inverse Probl. Imaging 1, 691$712(2007)$

39. Serranho, P.: A hybrid method for inverse obstacle scattering problems. Dissertation Göttingen (2007)

40. Wienert, L.: Die numerische Approximation von Randintegraloperatoren für die Helmholtzgleichung im $\mathbb{R}^{3}$. Dissertation Göttingen (1990) 